\title{
NASA-CR-204717 Latitudinal dependence of the radial IMF component: Coronal imprint
}

\author{
S. T. Suess \\ NASA Marshall Space Flight Center, Huntsville, Alabama \\ E. J. Smith \\ Jel Propulsion Laboratory, Pasadena, California
}

\begin{abstract}
Measurements by Ulysses have confirmed that there is no significant gradient with respect to heliomagnetic latitude in the radial component, $B_{r}$, of the interplanetary magnetic field. In the corona, the plasma $\beta$ is $<<1$, except directly above streamers, so longitudinal and latitudinal gradients in field strength will relax due to the transverse magnetic pressure gradient force as the solar wind carries magnetic flux away from the Sun. This happens quickly enough so that the field is essentially uniform by $5-10 R_{\odot}$, apparently remaining so as it is carried to beyond $1 \mathrm{AU}$. Here, we illustrate the coronal relaxation with a qualitative physical argument and by reference to a detailed MHD simulation.
\end{abstract}

\section{Introduction}

Ulysses in 1993-1995 [Smith and Balogh, 1995; Balogh et al., 1995] and ICE and IMP-8 in 1984-1988 [Burton et al., 1995] observed no significant gradient in heliomagnetic latitude in the radial component, $B_{r}$, of the interplanetary magnetic field (IMF) at heliocentric distances of 1-4 AU. These observations were made near solar minimum when the Sun's magnetic field is most nearly an axially aligned dipole. Figure 1 shows the results from IMP-8 and ICE scaled to $1 \mathrm{AU}$ and plotted against magnetic latitude. The data are five degree bin averages. Curves (a), (b), and (c) are from models which will be discussed below. The apparent small gradient in $B_{r}$ near the magnetic equator is probably due to small errors in sector identification so that the true gradient is completely negligible. Essentially no gradient was observed by Ulysses in 1993-1995 up to a heliographic latitude of $80^{\circ}$. Here we discuss the reason why the magnetic field at the top of the corona, i.e. at $10 R_{\odot}$, does not have any significant gradient in latitude or longitude outside of the heliospheric plasma sheet (HPS) [Gosling et al., 1981] surrounding the heliospheric current sheet (HCS). In $\S 2$, we explain why

Copyright 1996 by the American Geophysical Union.

Paper number $96 \mathrm{GL} 02908$. 0094-8534/96/96GL-02908\$05.00 the coronal imprint on the IMF lacks latitudinal and longitudinal (i.e. $(\theta, \phi))$ gradients on a physical basis. This is verified in Section 3 using results from an MHD model of the corona. In a second study, we examine the absence of further redistribution of magnetic flux in the interplanetary medium by latitudinal gradients in thermal pressure [Suess et al., 1996].

\section{The Coronal Magnetic Field}

The immediate reason for the absence of $(\theta, \phi)$ gradients in the magnetic field at $10 R_{\odot}$ is that the plasma $\beta$ (the thermal pressure divided by the magnetic pressure) is small between $1.0 R_{\odot}$ and $\sim 10.0 R_{\odot}$ except in the HPS above streamers. This is demonstrated here using a potential field-source surface (PFSS) model of coronal structure as a tool. PFSS models are routinely used to estimate coronal streamer locations and sizes and the location of the magnetic neutral line lying above the streamer belt, which defines the base of the heliospheric current sheet (HCS) [Hoeksema, 1991]. However, they fail to predict the absence of $(\theta, \phi)$ gradients in the interplanetary medium. In spite of this failure, a PFSS model can show that the gradients are smoothed out over a very short distance inside $10 R_{\odot}$. This is done by using the PFSS model to demonstrate its own internal inconsistency and to estimate the speed at which the gradients are therefore smoothed.

In a PFSS model the field is assumed to be current free between the photosphere and a "source surface" placed at about $2.5 R_{\odot}$, the source surface is assumed spherical, and the magnetic field is defined to be radial at the source surface. The boundary condition at $1.0 R_{\odot}$ is that a scalar be specified over the sphere - normally the observed line-of-sight field. Figure 2 shows the line of sight photospheric field and the resulting source surface magnetic field in a PFSS model using Wilcox Solar Observatory data for Carrington Rotation 1779 (August-September 1986) [Hoeksema, 1991]. This rotation is included in the time period of IMP- 8 and ICE data used in Figure 1, and it is obvious the source surface field varies smoothly up to and across the neutral line, unlike the IMF which exhibits a discontinuity at the HCS. The implied transverse gradients in $\left|\mathbf{B}^{2}\right|$, $\partial\left|\mathbf{B}^{2}\right| / \partial \phi$ and $\partial\left|\mathbf{B}^{2}\right| / \partial \theta$, which are completely 


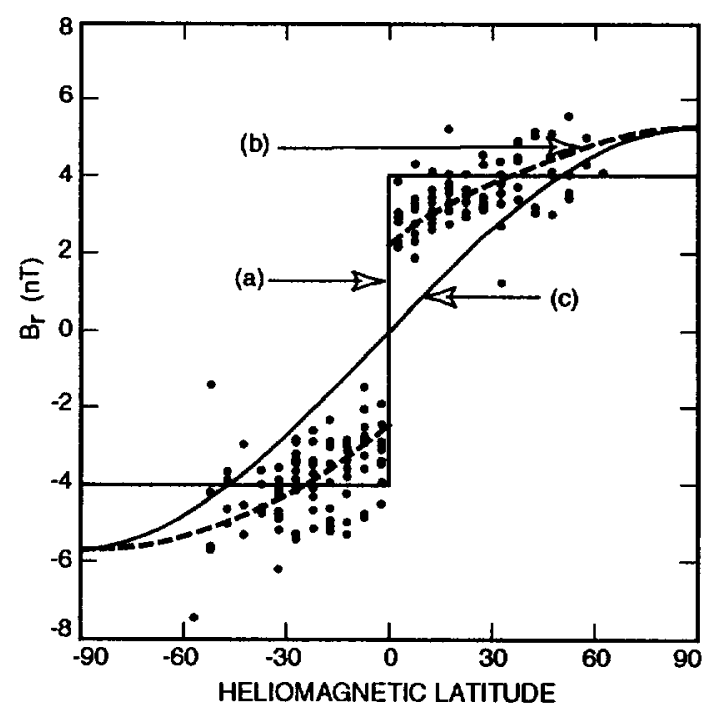

Figure 1. Radial magnetic field values in five degree latitude bins, scaled to $1 \mathrm{AU}$, from ICE and IMP-8 during 1984-1988, bracketing solar minimum [Burton et al. 1995]. (a) is a pure current sheet model [Wolfson, 1985], at a heliocentric distance of more than $50 R_{\odot}$. (b) is from Pneuman and Kopp's [1971] MHD model, at a large distance from the base. (c) is the field variation for a potential field source surface model with the source surface at $2.5 R_{\odot}$. inconsistent with the observations shown in Figure 1, would have to be balanced by corresponding gradients in the pressure to persist.

The distance it will take for the gradients to relax can be calculated using the PFSS model to estimate the relaxation speed. Assume the coronal magnetic field between $1 R_{\odot}$ and the source surface at $R_{s}$ to be defined by

$$
\mathbf{B}=-\nabla \Phi \quad \nabla^{2} \Phi=0
$$

Taking the field to be radial on $R_{s}$, axisymmetric, and dipolar at $1 R_{\odot}$ where $B_{r}\left(R_{\odot}\right) \equiv B_{r o} \cos \theta=$ $B_{\text {ro }} P_{1}^{0}(\cos \theta)$ gives

$$
\Phi(r, \theta, \phi)=\frac{B_{r o} R_{\odot} \cos \theta}{2+\left(R_{\odot} / R_{s}\right)^{3}}\left[\left(\frac{R_{\odot}}{r}\right)^{2}-\frac{r R_{\odot}^{2}}{R_{s}^{3}}\right]
$$

A plot of the field lines from (2) is shown in Figure 3. The solid lines are field lines derived from (2) and the dashed lines are for a vacuum dipole in which all of the field lines return to the base. For the physical parameters, we use the observed radial field strength at $1 \mathrm{AU}$ of $3.5 \times 10^{-5} \mathrm{G}$ [Smith and Balogh, 1995] and extrapolate it back to the Sun to give $B_{r o}=4.4 \mathrm{G}$. For the temperature and density, let $T\left(R_{s}\right)=10^{6} \mathrm{~K}$ and $n\left(R_{s}\right)=3 \times 10^{5} \mathrm{~cm}^{-3}$ [Allen, 1964; Habbal et al.,
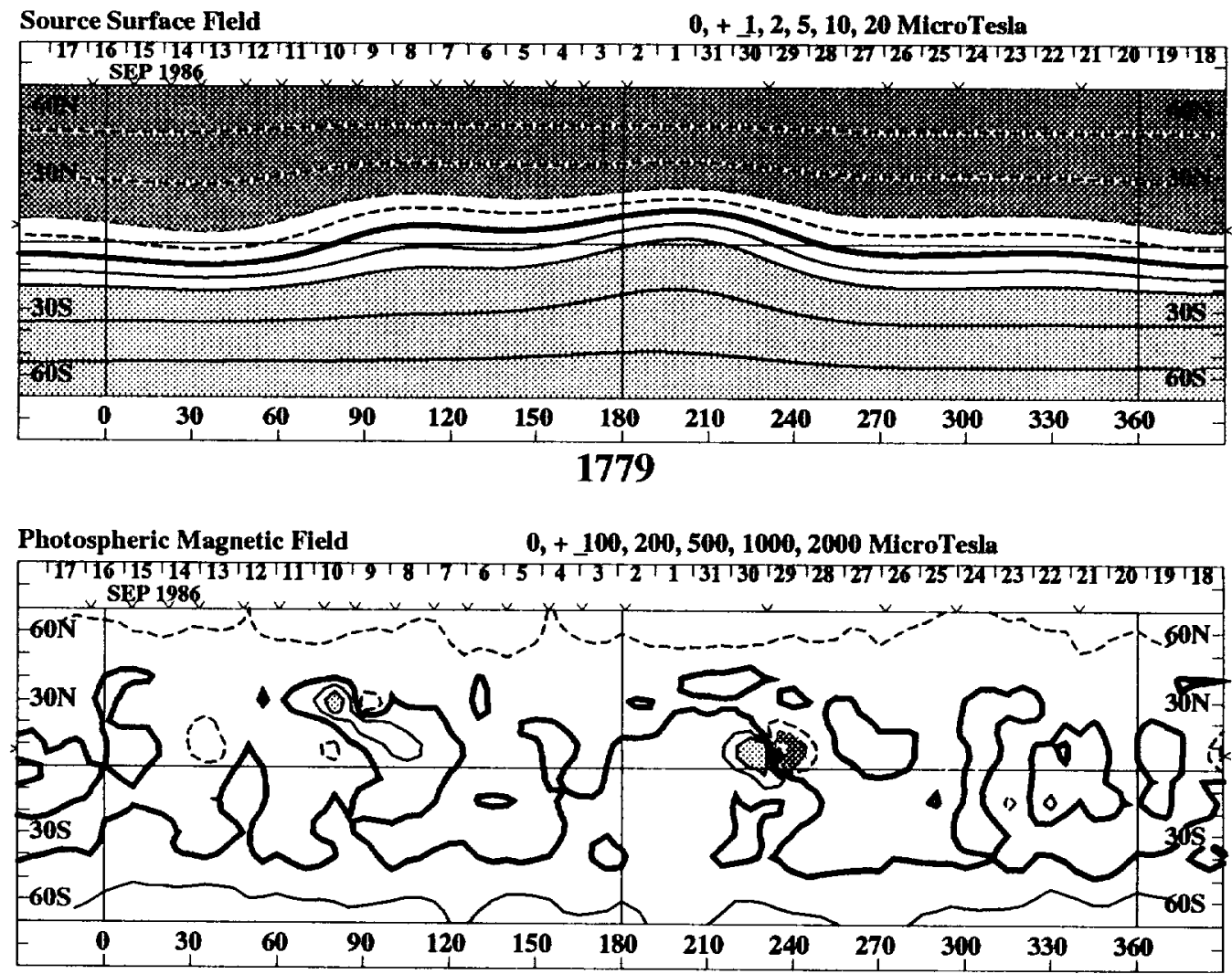

1779

Figure 2. Synoptic charts of the photospheric (bottom) and computed coronal (top) magnetic fields in September 1986 - Carrington Rotation 1779. The heavy solid line shows the neutral line dividing inward and outward polarity regions. The dashed contours and heavy shading show negative (inward) field regions. Solid contours and light shading show positive areas. Shading begins at $\pm 200 \mu \mathrm{T}$ at the source surface located at $2.5 R_{\odot}$. No data above $70^{\circ}$ are shown because they are not measured independently due to the low spatial resolution of the Wilcox Solar Observatory observations [Hoeksema, 1991]. 


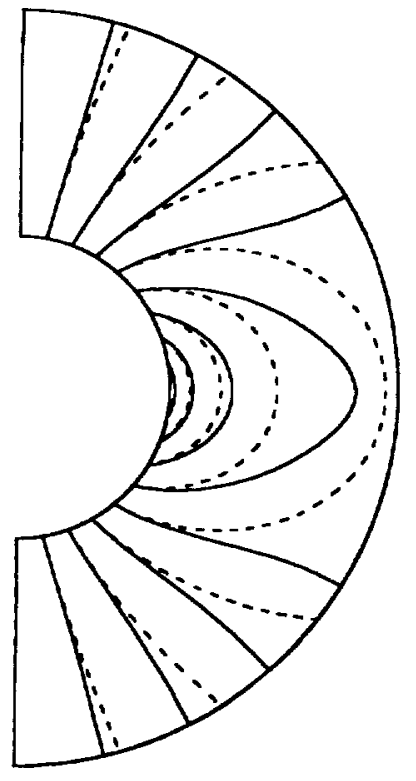

Figure 3. Magnetic field lines for a vacuum dipole (dashed lines) and a PFSS model with the source surface placed at $2.5 R_{\odot}$ (solid lines) in which the boundary condition on the source surface is that the field be radial. The source surface is at the outer half-circle.

1995]. Then, with $\beta \equiv 2 n k T /\left(B_{r}^{2} / 8 \pi\right)$, evaluation on the source surface gives

$$
\begin{gathered}
B_{r}\left(R_{s}, \theta\right)=0.4 \cos \theta \quad \mathrm{G} \\
\beta\left(\theta, R_{s}\right)=0.0126 \cos ^{-2} \theta
\end{gathered}
$$

such that $\beta\left(\theta=0, R_{s}\right)=0.0126$ and $\beta\left(\theta=80^{\circ}, R_{s}\right)=$ 0.42 . Curve (c) in Figure 1 shows equation (2) extrapolated to $1 \mathrm{AU}$ and compared with the observed field, again illustrating the failure of the PFSS model to predict the observed variation. Equation (3) shows that for typical parameters, $\beta<1$ over the bulk of the open region. Only directly over the streamer is $\beta \geq 1$, asymptotically approaching $\infty$ as $\theta \rightarrow 90^{\circ}$. Inside the source surface, $\beta$ decreases further, while it grows only slowly until it becomes of $\mathrm{O}[1]$ only in the vicinity of $10 R_{\odot}$. The implied transverse magnetic pressure imbalance cannot be sustained by thermal pressure gradients and will therefore be reduced, or relax, in the solar wind frame of reference. This occurs approximately at the fast mode speed, $v_{f}$, because the magnetic pressure gradients and the consequent relaxation are perpendicular to the direction of the magnetic field.

The fast mode speed is given by

$$
v_{f}^{2}(\theta)=\frac{2 k T}{m_{p}}+\frac{B_{r}^{2}\left(R_{s}, \theta\right)}{4 \pi n m_{p}}
$$

such that $v_{f}(\theta) \approx 1620 \cos \theta \mathrm{km} / \mathrm{s}$. Assume for this example that the radial flow speed of the solar wind in the mid-corona is $v_{r}=400 \mathrm{~km} / \mathrm{s}$. This is probably large compared to typical coronal flow speeds and therefore will give an upper bound to the relaxation distance, $\lambda_{r}$. For a tranverse relaxation through a latitude angle $\left(\theta-\theta_{s}\right)$, at speed $v_{f}$, in solar wind moving at speed $v_{r}$, $\lambda_{r}$ can be estimated by using the angular speed given by $\dot{\theta}=\left(v_{f} / r\right)=d \theta / d t$. Substituting $v_{r}=d r / d t$ and integrating gives

$$
\begin{aligned}
& d \theta=\left(v_{r} / r\right) d t=\left(v_{f} / r\right)\left(d r / v_{r}\right) \\
& \theta-\theta_{s}=\left(v_{f} / v_{r}\right) \ln \left(r / R_{s}\right)
\end{aligned}
$$

where, $\lambda_{r}=\left(r-R_{s}\right) . \theta_{s}$ is the starting angle at $R_{s}$. Taking $\left(\theta-\theta_{s}\right)=45^{\circ}=\pi / 4$ radians to represent a large transverse angular displacement and assuming a nominal mean value for $v_{f}$ of $1200 \mathrm{~km} / \mathrm{s}$, then $\lambda_{r}=$ $0.3 R_{s}=0.75 R_{\odot}$. The relaxation is therefore complete inside $5 R_{\odot}$. This could have been anticipated because $v_{f} \gg v_{r}$ in this region. So, rather than (2) representing the coronal imprint on the IMF radial magnetic field strength, it is appropriate to take the field strength as constant at the top of the corona everywhere except in the HPS.

\section{Comparison With An MHD Model}

The above argument based on the fast mode wave speed in the corona is fully supported by self-consistent MHD calculations of the relaxation of the transverse gradients [e.g., Suess, et al. 1977, Fig. 2; Steinolfson, Suess, and Wu, 1982; Steinolfson, 1988]. The results from the earliest such calculation [Pneuman and Kopp, 1971] are shown by curve (b) in Figure 1, where there is a large, but not complete relaxation in the transverse field gradients - probably being incomplete due to the photospheric field strength in this calculation being only $1 \mathrm{G}$. A full relaxation is explicitly shown in the quantitative global coronal model of Wang, Wu, Suess, and Poletto [1995] which we use here as an example. The magnetic field variation with polar angle for the steady state is shown in Figure 4. This model has a steady volumetric heat source that varies with radius and polar angle, being smaller inside the equatorial streamer than in the polar coronal hole, and a field strength at the base of $\mathrm{O}[3] \mathrm{G}$. It produces densities in the hole and streamer of the same magnitudes as observed. The location of the streamer and the HPS lying above the streamer, where $\beta \geq 1$, are shown as the shaded region in Figure 4. This figure explicitly shows that the field strength is already nearly constant by $2.47 R_{\odot}$, and by $4.88 R_{\odot}$ the variation is insignificant - outside the HPS. This result is a detailed confirmation of the estimates made in $\S 2$ and closely reproduces the observations shown in Figure 1. The model further shows that $\beta \ll 1$ everywhere inside $\sim 10 R_{\odot}$ except in the HPS. Beyond $\sim 10 R_{\odot}, \beta \geq 1$.

Returning to PFSS models, these qualitatively simulate the field strength variation low in the corona, inside $\sim 2.0 R_{\odot}$. Conversely, a simple current sheet model of the corona leads to a uniform field strength far from the Sun as shown by curve (b) in Figure 1 [Wolfson, 1985]. Combining external current sheets with PFSS models has been successful in simulating the dynamics of the relaxation of the transverse magnetic field gradients [Zhao and Hoeksema, 1995; Wang and Sheeley, 1995] and is a way of retaining the practical applications 


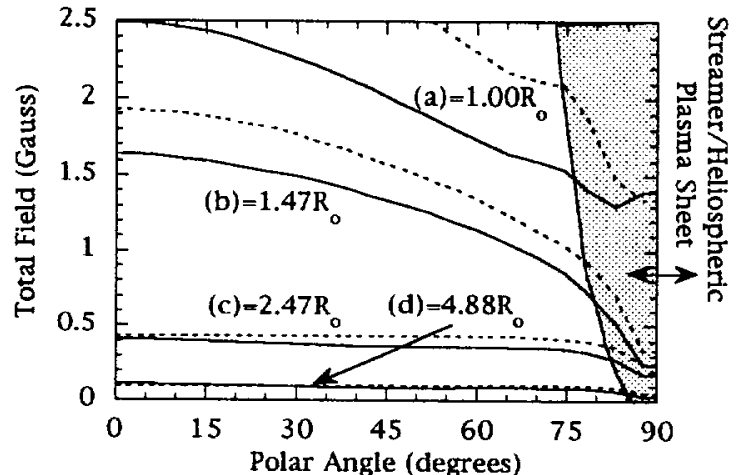

Figure 4. The total magnetic field strength at: (a) $1.00 R_{\odot}$, (b) $1.47 R_{\odot}$, (c) $2.47 R_{\odot}$, (d) $4.88 R_{\odot}$ versus polar angle. These values are taken from the global coronal simulation of Wang, Wu, Suess, and Poletto [1995] in which there is a volumetric heat source that produces a realistically thin heliospheric plasma sheet. The solid lines are the model described here.

of these relatively simple models. However, it must be recognized that they do not do a good job of modeling the transverse variation of the field strength between 2.0 and $10.0 R_{\odot}$.

Finally, the Ulysses, ICE, and IMP-8 results imply that latitudinal thermal pressure gradients are insufficient to redistribute magnetic flux in the interplanetary medium between $10 R_{\odot}$ and 1-4 $\mathrm{AU}$. We have analyzed the latitudinal temperature and density gradients observed at Ulysses and combined this with an analytic calculation of the resulting flux redistribution to show that an approximately constant field strength at $1 \mathrm{AU}$ is consistent with the observed pressure gradients [Suess et al., 1996].

Acknowledgments. This work has been supported by the NASA Ulysses Project and by the Jet Propulsion Laboratory, California Institute of Technology, under contract with the National Aeronautics and Space Administration.

\section{References}

Allen, C. W., Astrophysical Quantities, 2nd ed., The Athlone Press, University of London, 1964.

Balogh, A., E. J. Smith, B. T. Tsurutani, D. J. Southwood, R. J. Forsyth, and T. S. Horbury, The heliospheric magnetic field over the south polar region of the Sun, Science, 268, 1007-1010, 1995.
Burton, M. E., E. J. Smith, A. Balogh, and N. Murphy, Heliomagnetic latitude dependence of the heliospheric magnetic field, proc. of Solar Wind 8 (D. Winterhalter, D. McComas, N. Murphy, and J. Phillips, eds.), in press, 1995.

Forsyth, R. J., The high latitude heliospheric magnetic field, Space Sci. Rev., 72, 153-163, 1995.

Gosling, J. T., G. Borrini, J. R. Asbridge, S. J. Bame, W. C. Feldman, and R. T. Hansen, Coronal streamers in the solar wind at 1 AU, J. Geophys. Res., 86, 5438-5448, 1981.

Habbal, S. R., R. Esser, M. Guhathakurta, and R. R. Fisher, Flow properties of the solar wind derived from a two-fluid model with constraints from white light and in situ interplanetary observations, Geophys. Res. Lett., 22, 1465, 1995.

Hoeksema, J. T., Large-scale solar and heliospheric magnetic fields, Adv. Space Res., 11, 15-24, 1991.

Pneuman, G., and R. A. Kopp, Gas-magnetic field interactions in the solar corona, Solar Phys., 71, 361-379, 1971.

Smith, E. J., and A. Balogh, Ulysses observations of the radial magnetic field, Geophys. Res. Lett., 22, 3317, 1995.

Steinolfson, R. S., Density and white-light brightness in looplike coronal mass ejections: Importance of the preevent atmosphere, J. Geophys. Res., 93, 14,261, 1988.

Steinolfson, R. S., S. T. Suess, and S. T. Wu, The steady global corona, Astrophys. J., 255, 730-742, 1982.

Suess, S. T., A. K. Richter, C. R. Winge, and S. F. Nerney, Solar polar coronal hole - a mathematical simulation, $A s$ trophys. J., 217, 296-305, 1977.

Suess, S. T., E. J. Smith, J. Phillips, B. E. Goldstein, and S. Nerney, Latitudinal dependence of the radial IMF component - interplanetary imprint, Astron. Astrophys., in press, 1996.

Wang, Y.-M., and N. R. Sheeley, Jr., Solar implications of the Ulysses interplanetary field measurements, Astrophys. $J$. Lett., 447, L143-L146, 1995.

Wang, A.-H., S. T. Wu, S. T. Suess, and G. Poletto, Numerical modeling of coronal mass ejections based on various pre-event model atmospheres, Solar Phys., 161, 365-381, 1995.

Wolfson, R., A coronal magnetic field model with volume and sheet currents, Astrophys. J., 288, 769, 1985.

Zhao, X.-P., and J. T. Hoeksema, Prediction of the interplanetary magnetic field strength, J. Geophys. Res., 100, 19-33, 1995.

E. J. Smith, Jet Propulsion Laboratory, California Institute of Technology, Pasadena, California 91109-8099.

S. T. Suess, NASA Marshall Space Flight Center, Mail Stop 82, Huntsville, AL 35812.

(Received November 14, 1995; revised June 17, 1996; accepted June 21, 1996.) 